\title{
River bedform inception by flow unsteadiness: A modal and nonmodal analysis
}

\author{
Alice Caruso, ${ }^{1,}{ }^{*}$ Riccardo Vesipa,${ }^{1}$ Carlo Camporeale, ${ }^{1}$ Luca Ridolfi, ${ }^{1}$ and Peter J. Schmid ${ }^{2}$ \\ ${ }^{1}$ Department of Environmental, Land and Infrastructure Engineering, Politecnico di Torino, Corso Duca Abruzzi 24, 10129 Turin, Italy \\ ${ }^{2}$ Department of Mathematics, Imperial College London, London SW7 2AZ, United Kingdom
}

(Received 25 January 2016; published 17 May 2016)

\begin{abstract}
River bedforms arise as a result of morphological instabilities of the stream-sediment interface. Dunes and antidunes constitute the most typical patterns, and their occurrence and dynamics are relevant for a number of engineering and environmental applications. Although flow variability is a typical feature of all rivers, the bedform-triggering morphological instabilities have generally been studied under the assumption of a constant flow rate. In order to partially address this shortcoming, we here discuss the influence of (periodic) flow unsteadiness on bedform inception. To this end, our recent one-dimensional validated model coupling Dressler's equations with a refined mechanistic sediment transport formulation is adopted, and both the asymptotic and transient dynamics are investigated by modal and nonmodal analyses.
\end{abstract}

DOI: 10.1103/PhysRevE.93.053110

\section{INTRODUCTION}

River bedforms are widespread morphological patterns which arise as a result of an unstable interaction between a turbulent free-surface flow and an erodible bottom. Research interest about these morphologies derives from their relevance in hydraulic engineering and environmental applications. River bedforms not only interfere with river navigation $[1,2]$ and human infrastructure $[3,4]$, they also induce differential pressure gradients that modify the flow field and consequently the overall hydraulic resistance, induce hyporheic fluxes [5,6], and affect underground flows through preferential patterns within ancient sedimentary deposits $[7,8]$. The present work focuses on dunes and antidunes (see Fig. 1), which are microscale patterns generated under subcritical (Froude number, $\left.F_{0}<1\right)$ and supercritical $\left(F_{0}>1\right)$ streams, respectively. Whereas dunes propagate in the downstream direction, antidunes are characterized by a periodic pattern that migrates upstream and causes the free surface to be in phase with the bottom.

Dunes exhibit different shapes, depending on the amount of available erodible sediments and on the width-to-depth ratio of the river. In this work, we focus on two-dimensional transverse dunes and antidunes, which arise perpendicularly to the flow direction in narrow rivers. However, it is worth recalling that transverse dunes can decay into a chain of barchans (crescent-shaped dunes) and display a "sea-wave-like" shape with meandering. The formation of barchan dunes is very common and widely studied in an aeolian environment, as demonstrated by a vast body of literature [9-11], but has also been detected in subaqueous conditions both in laboratory experiments [12] and in real rivers [13].

Traditionally, the stability analysis of the sediment-fluid interface has largely been studied using two important simplifications: (i) the use of classical linear stability theory based on normal modes and (ii) the hypothesis of steady base conditions, i.e., no discharge variations [15-20]. The modal (or normal) approach constitutes a powerful mathematical tool in stability theory and has been extensively used in

*alice.caruso@polito.it fluid mechanics for more than a century. By means of this analysis, the dispersion relationship that relates the growth rate of the disturbance to its wave number vector is obtained. A zero-growth-rate condition allows one to obtain the neutral (marginal) stability condition as a function of the governing parameters and to delineate stability and instability regions in parameter space. The main goal of such an approach is to establish the asymptotic temporal fate of the disturbances (i.e., for $t \rightarrow \infty$ ), since focus is on the least stable eigenvalue. In this manner, one determines whether disturbances tend to zero or infinity as time tends to infinity and, accordingly, classifies the base state as asymptotically stable or unstable. However, no information is gained on the disturbance behavior over finite time horizons; in particular, the stability of the system is determined, regardless of the way in which the disturbance tends to zero.

In order to understand the importance of studying the system behavior over finite times as well, three emblematic qualitative temporal evolutions of perturbations are represented in Fig. 2(a). The analysis of asymptotic behavior by means of eigenvalues allows the asymptotically stable cases $\mathrm{A}$ and $\mathrm{B}$ (the perturbation decays to zero for $t \rightarrow \infty$ ) to be distinguished from the unstable case $\mathrm{C}$, where the disturbance grows exponentially. However, curves A and B exhibit very different behavior for finite times: the perturbation in system A decays monotonically to zero, whereas it shows transient amplification in system B. The mathematical reason for this nonmonotonic behavior lies in the non-normality of the differential (or algebraic) operator which governs the temporal evolution of perturbations. This aspect is illustrated in Fig. 2(b) for a simple two-dimensional algebraic problem. The nonorthogonality of the eigenvector set implies that their superposition exhibits transient growth, even though both associated eigenvalues are negative and individual eigenvectors decay monotonically in time. As disturbances can be written as linear combinations of eigenvectors, nonorthogonality causes disturbances to experience transient growth similar to case B of Fig. 2(a), and the stronger the non-normality, the more pronounced the transient amplification. This short-term growth of the initial disturbance can be observed and investigated within a linear framework; no nonlinear mechanism needs to be invoked. 

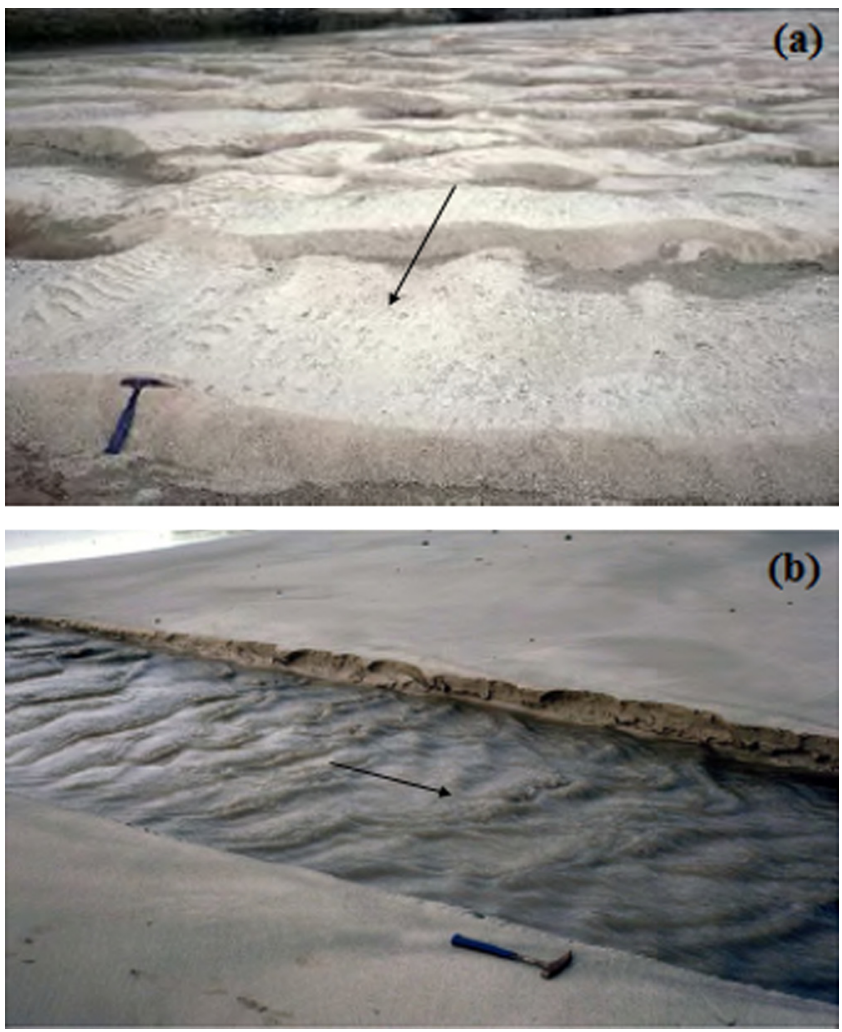

FIG. 1. Examples of morphologies relevant for the evolution of the river bottom: (a) river dunes after a flood event and (b) active antidunes [14]. The arrows indicate the stream direction. Bedform wavelength and amplitude are about $0.5-1 \mathrm{~m}$ and $10-20 \mathrm{~cm}$, respectively.

The distinction between monotonic and nonmonotonic temporal perturbation dynamics is not a mere mathematical detail, but is fundamental for several reasons. First, transient growth may trigger nonlinear instabilities. Thus, although the problem would be asymptotically stable according to a normalmode analysis, it could trigger transient instabilities which amplify (linearly) to such an extent that they render nonlinear terms significant (e.g., the dashed line in Fig. 2 is exceeded). This process-referred to as bypass transition [22,23]- has been suggested as a critical factor in shear-flow transition to account for discrepancies between experimental findings and analytical forecasts based on normal modes [24]. The second key point is that transient growth can occur on time scales that are comparable to those of the studied process. As a result, the system appears unstable, even though disturbances decay over far longer time scales. Third, the characteristics of transient growth (time scales, local growth rate, etc.) may strongly depend on the physical parameters as well as the characteristics of the disturbances. It is altogether conceivable that, over the first stages of evolution, perturbations that are most amplified differ in shape and wavelengths from the characteristics of the asymptotically most amplified solutions.

Nonmodal analysis of non-normal operators has a long tradition in fluid mechanics, providing insight into hydrodynamic instabilities for a variety of shear flows [24-26]. This approach has elucidated the role played by linear mechanisms in the triggering of instabilities in simple shear flows (e.g., [27-29]). (a)

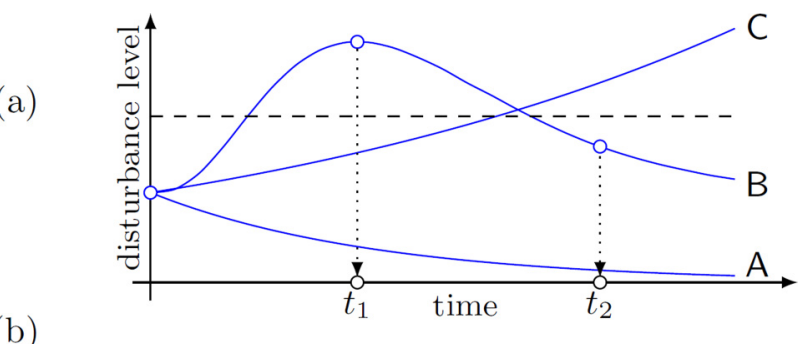

(b)

$\mathbf{e}_{2}$

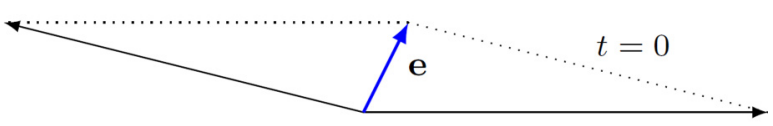

$\mathbf{e}_{2}$

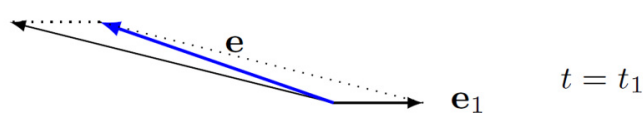

FIG. 2. Three possible linear evolutions of an initial, infinitesimal perturbation are reported in (a) monotonic asymptotic decay (A), transient growth before asymptotic decay (B), and monotonic indefinite growth $(\mathrm{C})$. The dashed line indicates a schematic possible threshold of the perturbation amplitude beyond which nonlinear terms start to be non-negligible. (b) illustrates the concept of transient amplification on a two-dimensional system, where the nonorthogonality of the two eigenvectors $\mathbf{e}_{1,2}$ gives rise to transient growth: even though both eigenvectors, $\mathbf{e}_{1}$ and $\mathbf{e}_{2}$, individually decay in time, their nonorthogonal superposition causes the norm (length) of the resulting vector $\mathbf{e}$ to exceed its initial value before ultimately decaying to zero. Figures are qualitative and adapted from [21].

In contrast, in a morphodynamic context this approach has only recently been applied, where it demonstrated the potential for transient growth for one-dimensional bed waves [30], river dunes [31], and bars [32]. Moreover, the transient dynamics of river patterns has not been investigated in the presence of flow variations. Even though unsteadiness is prevalent in any fluvial system, there are only a few studies on the effect of base-flow unsteadiness on the formation of morphodynamic instabilities [33-35], and an analysis of the interplay between unsteadiness and non-normality in the formation of river patterns is still missing.

The main aim of this study is to investigate the role that unsteady discharges play in the inception and subsequent development of river bedforms. To this end, we perform both modal and nonmodal analyses and study the stability of the system at short and long times. The classical stability analysis (by normal modes) is complemented by a nonmodal analysis. This allows us to uncover the existence of transient, short-time growth that is not predicted by the classic modal analysis. Therefore, the stability of the system is investigated across all inherent time scales. We use a depth-averaged model proposed by three of the authors [36]. This model was obtained by coupling one-dimensional shallow-water (Dressler) equations to a mechanistic sediment-transport formulation; it was validated with experimental data. The analysis will be limited to the case of temporally periodic variations of the flow discharge. In this way - and despite the simplicity of the underlying model-we will shed new light on river morphodynamics by addressing the mutual interaction between unsteadiness and non-normality. 


\section{PROBLEM FORMULATION AND GOVERNING EQUATIONS}

Let us consider a free-surface turbulent water stream flowing in a rectangular channel of width $B_{0}^{*}$ and slope $J$ (asterisked variables refer to dimensional quantities). The bed is composed of granular material with a mean grain diameter $d_{s}^{*}$. We define a local reference frame $\left\{s^{*}, n^{*}\right\}$ and a global Cartesian system $\left\{x^{*}, y^{*}\right\}$, linked by the relation $\partial / \partial s=\gamma \partial / \partial x$, with $\gamma=\cos \alpha$ and $\alpha$ denoting the local slope of the bed. The variables $\eta^{*}$ and $H^{*}$ indicate the local bed elevation and the free-surface elevation, respectively, while $D^{*}=H^{*}-\eta^{*}$ represents the local stream depth (see Fig. 3).

The base flow (unperturbed condition) is assumed unsteady with water depth $D_{0}^{*}(t)$ and longitudinal velocity $U_{0}^{*}(t)$. The following analysis considers the case of periodic time variations of the flow discharge in the form $Q^{*}\left(s^{*}, t^{*}\right)=$ $\bar{Q}_{0}^{*}+\delta^{*} \sin \left(\omega^{*} t^{*}+\phi\right)$, where $\bar{Q}_{0}^{*}$ stands for the mean value, $\delta^{*}$ represents the amplitude of the harmonic variation, $\omega^{*}$ denotes the angular velocity, and $\phi$ refers to the phase. The period of oscillation is given as $T^{*}=2 \pi / \omega^{*}$.

We consider the one-dimensional model proposed by Vesipa et al. [36], which allows analytical tractability of the full stability analysis. Two-dimensional approaches, where the dependence of the flow field on the vertical coordinate direction is maintained, require sophisticated numerical methods [37] and analytical techniques are precluded. The herein adopted one-dimensional depth-averaged flow model is based on two key elements which play a crucial role in the one-dimensional modeling of river-bed instabilities. The first element is the nonhydrostatic pressure induced by the curvature of the bottom. This pressure plays a pivotal role in correctly selecting the dominant wavelength of antidunes [36]. In fact, neglecting the bed curvature-induced nonhydrostatic pressure component still results in the prediction of an instability; the most unstable wavelength, however, is misrepresented. The second key element is the nonequilibrium modeling of the sediment transport. For this, we remove the hypothesis of uniform equilibrium conditions (i.e., the particle deposition rate equals the erosion rate in any point of the bed) on which many empirical formulas derived from experiments are based (e.g., the Fernandez-Luque and Van Beek formula). Conversely, a more refined mechanistic approach based on the momentum exchange between the fluid and the sediment and on the (space- and time-dependent) balance of the forces acting on the sediment particles $[38,39]$ is adopted. This approach allows

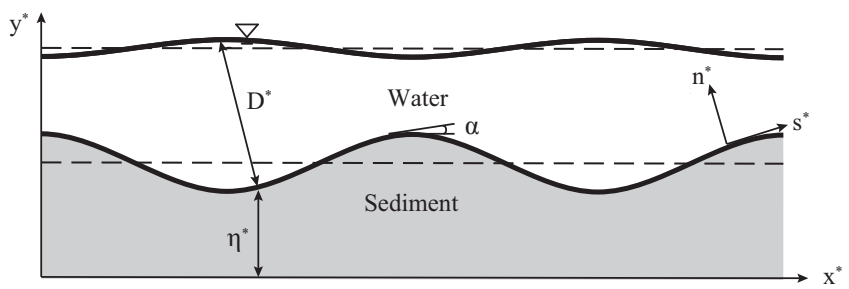

FIG. 3. Scheme of a channel with river bedforms. The dotted and continuous lines refer to unperturbed and perturbed conditions, respectively. Note that the free-surface elevation is given by $H^{*}-$ $D^{*}(1-\gamma)$ us to describe the unstable conditions more realistically, as the stream-bed system is far from a local equilibrium.

Let us briefly recall the main ingredients of the modeling approach, where the averaged values of depth, $\bar{D}_{0}^{*}=$ $\int_{0}^{T^{*}} D_{0}^{*}(t) d t / T^{*}$, and velocity, $\bar{U}_{0}^{*}=\int_{0}^{T^{*}} U_{0}^{*}(t) d t / T^{*}$, will be used to scale the governing quantities. Time is made dimensionless by the hydrodynamic temporal scale, namely, $t=t^{*} \bar{U}_{0}^{*} / \bar{D}_{0}^{*}$ and $\omega=\omega^{*} \bar{D}_{0}^{*} / \bar{U}_{0}^{*}$, where $\omega$ stands for the dimensionless angular velocity. The hydrodynamics are described by the one-dimensional Dressler formulation [40] that generalizes the classical Saint-Venant equations to the case of a nonhydrostatic pressure distribution, therefore allowing us to also consider the profile of normal velocity. The continuity equation and the longitudinal momentum equation are given as

$$
\begin{aligned}
& \frac{\partial D}{\partial t}+\frac{U}{\mathcal{N}^{2}} \frac{\partial D}{\partial s}-V=0 \\
& \frac{\partial U}{\partial t}+\frac{U}{\mathcal{N}^{2}} \frac{\partial U}{\partial s}+\frac{\sin \alpha}{F_{0}^{2}}+\frac{\tau_{B}}{D(1-\kappa D / 2)}+\left.\frac{\partial \mathcal{P}}{\partial s}\right|_{n=0}=0
\end{aligned}
$$

where $\mathcal{N}=1-\kappa D$, with $\kappa$ as the local bed curvature, $D$ as the dimensionless depth measured perpendicular to the channel bed, and $V$ as the dimensionless normal velocity evaluated at $n=D$. Equation (2) accounts for the effects of flow acceleration (first two terms), gravity (third term), and the drag induced by bed roughness (fourth term). The shear stress at the bottom, $\tau_{B}$, is evaluated using the Chezy formula $\tau_{B}=C U^{2}$, where $C$ is the friction coefficient, which is a function of the relative roughness $d_{s}$ and the dimensionless depth, following a closure relation provided by [41]. We note that the dimensional flow shear velocity of the basic state can be obtained as $u_{0 *}=U_{0}^{*} \sqrt{C}$. The last term in (2) reads

$\left.\frac{\partial \mathcal{P}}{\partial s}\right|_{n=0}=\left(\frac{\gamma}{F_{0}^{2}}+\frac{\kappa U^{2}}{\mathcal{N}^{3}}\right) \frac{\partial D}{\partial s}-\left(\frac{\kappa \sin \alpha}{F_{0}^{2}}-\frac{U^{2}}{\mathcal{N}^{3}} \frac{\partial \kappa}{\partial s}\right) D$,

where $\mathcal{P}(s, n, t)$ is the vertical profile of the dimensionless pressure. Accordingly, the Froude number, defined as $F_{0}=$ $U_{0}^{*} /\left(g D_{0}^{*}\right)^{1 / 2}$ (with $g$ as the gravitational acceleration), is periodically dependent on time. A morphodynamic model which accounts for the morphological evolution of the river bed is coupled to the shallow-water formulation of hydrodynamics. Through a mechanistic approach, sediment deposition and erosion rates are not balanced, because the flow is nonuniform; the dimensionless sediment transport rate, $q$, is given by $q=\xi v$, where $v(s, t)$ denotes an ensemble average of the particle velocity and $\xi(s, t)$ represents the area-based particle concentration.

The competition between the local entrainment and deposition of particles provides the longitudinal gradient of the sediment transport, according to the following balance equation for the sediment mass:

$$
\frac{1}{Q_{h}} \frac{\partial \xi}{\partial t}+\frac{\partial q}{\partial x}=\Theta(\mathcal{E}-\mathcal{D}),
$$

where $\Theta=\left(R d_{s}^{3}\right)^{1 / 2} /(1-p) F_{0}=\mathcal{O}\left(10^{-3}-10^{-4}\right), p$ denotes the porosity, $R=\rho_{s} / \rho-1$ is the submerged sediment density, and $\rho_{s}$ and $\rho$ are the sediment and fluid densities, respectively. 
We select $p=0.4$ and $R=1.65$, which are typical values for silicate sediments $[17,30]$, but the choice of other reasonable values would not greatly modify the picture that emerges. $Q_{h}$ stands for the ratio between the scale of sediment discharge and the flow rate discharge. The sediment erosion rate $\mathcal{E}$ and the sediment deposition rate $\mathcal{D}$ can be accounted for via the relations proposed by [39]

$$
\begin{aligned}
\mathcal{E} & =\frac{r_{e} A_{e}}{d_{s}}\left(\theta_{f}-\theta_{c}\right)^{3 / 2}, \\
\mathcal{D} & =(1-p) \frac{r_{s} A_{s}}{d_{s}^{2}} \theta_{s}^{1 / 2} \xi,
\end{aligned}
$$

where $A_{e}=0.028, A_{s}=0.068$, and $r_{e}$ and $r_{s}$ are reported in the Appendix. As far as the total shear stress exerted on the bottom of the bed-load layer is concerned, two components have to be considered: the (dimensionless) stress exerted by the sediment, $\tau_{s}$, and the stress exerted by the fluid, $\tau_{f}$, where $\tau_{s}+\tau_{f} \sim \tau_{B}$ and $\theta=F_{0}^{2} \tau / R d_{s}$ is the Shields stress.

By expressing the stress exerted by the sediment as a quadratic function of the relative velocity of the particle with respect to the fluid, and imposing a standard velocity distribution near the bottom, the relationship between the different components of stress is as follows:

$$
\theta_{f}=\theta_{B}-T_{\mu} \xi\left(\frac{f}{F_{0}} \sqrt{R d_{s} \theta_{B}}-v\right)^{2},
$$

with $f=11.5$ and $T_{\mu}$ as a parameter of the mechanistic transport model that accounts for gravity effects. The expression for $T_{\mu}$ is reported in the Appendix, together with the parameters $R_{0}$ and $S_{0}$, introduced below. It has been established that gravity effects play a secondary role in triggering river-bed instabilities when compared to the stream-induced forces [36]. However, gravitational effects influencing transport thresholds are nonetheless maintained for a more rigorous treatment.

The longitudinal gradient of the sediment transport is responsible for the evolution of the bed elevation as established by the Exner equation, namely, by a kinematic condition for the bottom boundary which reads

$$
\frac{\partial \eta}{\partial t}+\frac{\partial(v \xi)}{\partial x}=0
$$

Finally, the particle velocity $v$ is evaluated using a dynamical balance of all forces acting on the sediment grain

$$
I^{*}=F^{*}-A^{*}-G_{\|}^{*},
$$

where $F^{*}$ is the drag force (proportional to $\left[f \sqrt{\theta_{B}}-q\right]^{2}$, as in (6)); $A^{*}=\mu_{d} G_{\perp}^{*}$ is the resistive force due to friction $\left(\mu_{d}\right.$ is the dynamic friction coefficient; see Appendix); $\left\{G_{\perp}^{*}, G_{\|}^{*}\right\}=$ $G^{*}\{\cos \alpha, \sin \alpha\}$ are the components of the submerged particle weight, respectively measured normal and parallel to the bottom; and $I^{*}$ is the particle inertia. In dimensionless form, we obtain

$$
\frac{\partial v}{\partial t}+v \frac{\partial v}{\partial x}+R_{\mu}-S_{\mu}\left(\frac{f}{F_{0}} \sqrt{R d_{s} \theta_{B}}-v\right)^{2}=0 .
$$

In summary, the five partial differential equations (1), (2), (4), (7), and (9) constitute our hydromorphodynamic model where the unknowns are $\mathbf{X}=\{U, H, \eta, v, \xi\}$, recalling that $D=H-\eta$.
In setting up a linear stability analysis, we perturb the unsteady base state with an infinitesimal periodic perturbation following the ansatz

$$
\mathbf{X}=\left\{U_{0}(t), H_{0}(t), \eta_{0}, v_{0}(t), \xi_{0}(t)\right\}+\varepsilon \mathbf{X}_{1} \mathrm{e}^{i k \mathrm{x}},
$$

where $\mathbf{X}_{1}=\left\{u_{1}(t), h_{1}(t), \eta_{1}(t), v_{1}(t), \xi_{1}(t)\right\}$ contains the amplitudes of the wavelike perturbations, $k$ denotes the longitudinal wave number, and $\varepsilon \ll 1$ is the amplitude of the bed perturbation. After substituting (10) into our model equations and expanding in $\varepsilon$, we can solve for the unperturbed basic state, obtaining analytical expressions for $\bar{U}_{0}, \bar{H}_{0}, \bar{v}_{0}$, and $\bar{\xi}_{0}$ as well as relations between the Froude number and the discharge. The first-order terms in the expansion, $\mathcal{O}(\varepsilon)$, yield a governing system of linear differential equations of the form

$$
\frac{d}{d t} \mathbf{X}_{1}(t)=\mathbf{A}(t) \mathbf{X}_{1}(t)
$$

where $\mathbf{A}(t)=\mathbf{A}(t+T)$ is a $5 \times 5$ time-periodic matrix with period $T=2 \pi / \omega$, which depends on the base-state variables and on the sediment and stream characteristics of the problem.

\section{MODAL ANALYSIS}

A modal analysis aims at the assessment of the asymptotic, long-term fate of infinitesimal perturbations superimposed on the base state. Using this framework, the stability of the periodic solution for long times can be investigated by Floquet theory, a technique which permits the analysis of linear systems with the time periodic basic state. Accordingly, the system is classified as asymptotically stable if all infinitesimal perturbation decays in time as $t \rightarrow \infty$; otherwise, the base system is unstable. Floquet stability analysis supplies the natural modes of the perturbation behavior and hence the general stability properties of the system's equilibrium. According to Floquet theory, the fundamental solution operator associated with (11) is introduced as the matrix $\Psi(t)$ which is computed by advancing a $5 \times 5$ identity matrix over one temporal period (from $t=0$ to $t=T$ ) according to (11). Each column of $\Psi(t)$ is a linearly independent solution of the system. The asymptotic stability of the problem is inferred from the eigenvalues $v_{j}$ (Floquet multipliers) of the monodromy matrix $\mathcal{B}$, defined as $\mathcal{B}=\Psi^{-1}(0) \Psi(T)$. The flow is deemed asymptotically stable, if all eigenvalues of $\mathcal{B}$ fall inside the unit disk, suggesting a contractive map over a full period; otherwise, the flow is asymptotically unstable.

\section{NONMODAL ANALYSIS}

Nonmodal analysis addresses the transient evolution of disturbances over finite times, taking into account the nonnormality of the governing operator. In this analysis, a key point is to find a suitable metric for the perturbation magnitude. Following the temporal evolution of the total fluctuation energy can be physically motivated and is commonplace in studies of transient behavior and non-normality [26,28]. For the present problem, the dimensionless energy is composed of a kinetic component $K=K_{u}+K_{v}$, where $K_{u}$ and $K_{v}$ are the contributions from fluctuations of flow and particle velocities, as well as a potential component $P=P_{s}+P_{b}$, where $P_{s}$ and $P_{\eta}$ account for the free-surface and bed fluctuations. The flow's 
kinetic energy is easily obtained as $K_{u}=\left|u_{1}\right|^{2} / 2$, while the kinetic energy of the solid particles, $K_{v}$, can be assumed to be proportional to the velocity $\left(\xi v / h_{s}\right)^{2}$, where $h_{s}$ is the bed-load layer thickness, considered equal to $2.5 d_{s}$ [38]. The product $\xi v$ is the volumetric solid discharge per unit width which, when divided by $h_{s}$, provides a particle velocity averaged over a moving volume. After expanding in Fourier series and integrating in $x$ over one wavelength, we obtain

$$
K_{v}=\frac{K\left(\xi_{0}^{2}\left|v_{1}\right|^{2}+v_{0}^{2}\left|\xi_{1}\right|^{2}\right)}{2}+2 K v_{0} \xi_{0}\left(v_{1} \xi_{1}^{H}+v_{1} \xi_{1}^{H}\right)
$$

with $K=0.4 \rho_{s}(1-p)^{2} /\left(2 \rho d_{s}\right)$. The superscript $H$ refers to the complex conjugate. The contributions $P_{s}$ and $P_{\eta}$ coincide with those reported in [30] neglecting the capillary forces (which is reasonable in a turbulent flow) and setting the null potential at the undisturbed surface and are equal to

$$
\begin{aligned}
& P_{s}=F_{0}^{-1} \frac{\left|h_{1}\right|^{2}}{2}, \\
& P_{\eta}=r F_{0}^{-1} \frac{\left|\eta_{1}\right|^{2}}{2},
\end{aligned}
$$

with $r=(1-p) R$. The last term of (12) causes the energy weight to be not positive definite, thus not complying with the definition of a norm. We must realize that the choice of an appropriate disturbance measure does not only stem from physical arguments but also must satisfy mathematical constraints. In order to arrive at a positive definite energy weight, while capturing a maximum of physical effects, we choose to neglect the last term of (12).

As the primary goal of this work is to analyze the morphological instabilities, our focus is on the behavior of the bed response. More specifically, we aim at determining initial conditions that maximize the transient growth of bed disturbances rather than maximize the global energy of the system at given times. This class of initial conditions can easily be identified by adding weight coefficients to the energy density such that the bed response is emphasized while the remaining components of energy are penalized - a technique that has previously been employed in [30,31]. The thus weighted energy is defined as follows:

$$
E=c K_{u}+c P_{s}+P_{\eta}+c K_{v},
$$

where $c$ is a coefficient much less than unit (we take $c=10^{-6}$; other values give similar results, provided that $c \ll 1)$. In this way, we consider a new kind of energy that formally uses all the components of the governing system (thus avoiding the issues of a seminorm [42]) but primarily consists of the bed potential energy. Provided that the vector $\mathbf{q}$ is defined as

$$
\mathbf{q}=\frac{\sqrt{2 c}}{2}\left\{u_{1}, \frac{h_{1}}{\sqrt{F_{0}}}, \eta_{1} \frac{\sqrt{r}}{\sqrt{c F_{0}}}, K \sqrt{\xi_{0}} v_{1}, K \sqrt{v_{0}} \xi_{1}\right\},
$$

the energy becomes simply $E=\|\mathbf{q}\|^{2}$, where $\|\cdot\|$ indicates the common $l_{2}$ norm. For this reason, Eq. (11) can be recast in terms of the disturbance energy, rather than the disturbance amplitude, as

$$
\frac{d}{d t} \mathbf{q}(t)=\mathbf{L}(t) \mathbf{q}(t)
$$

The nonmodal behavior of system (16) is investigated through the analysis of the growth function, which is defined as the maximum amplification of initial energy optimized over all admissible initial conditions [24], namely,

$$
\hat{G}(t)=\max _{\mathbf{q}_{0}} G(t)=\max _{\mathbf{q}_{0}} \frac{\|\mathbf{q}(t)\|^{2}}{\left\|\mathbf{q}_{0}\right\|^{2}},
$$

where $\mathbf{q}_{0}$ represents the initial disturbance, and $\|\cdot\|$ denotes the standard Euclidean 2-norm. Transient energy amplification occurs when $\hat{G}>1$.

For a time-dependent system matrix $\mathbf{L}(t)$, the problem can be solved by applying an optimization technique known as the direct-adjoint method [21,26], which determines the maximum amplification of initial energy that occurs over a specified time horizon. It involves maximizing a user-supplied cost functional and imposing constraints from the governing equations via Lagrange multipliers (or adjoint variables). The augmented cost functional is defined as

$$
\begin{aligned}
\mathcal{L}\left(\mathbf{q}, \tilde{\mathbf{q}}, \mathbf{q}_{0}, \tilde{\mathbf{q}}_{0}\right)= & \frac{\|\mathbf{q}(T)\|^{2}}{\left\|\mathbf{q}_{0}\right\|^{2}}-\int_{0}^{T}\left\langle\tilde{\mathbf{q}},\left[\frac{d}{d t}-\mathbf{L}\right] \mathbf{q}\right\rangle d t \\
& -\left\langle\tilde{\mathbf{q}}_{0},\left(\mathbf{q}(0)-\mathbf{q}_{0}\right)\right\rangle,
\end{aligned}
$$

where tildes indicate the Lagrange multipliers or adjoint variables. The first term on the right-hand side of (18) is the cost functional, while the second and third terms impose the constraints related to the governing equations and the initial conditions, respectively.

Seeking a maximum of the cost functional, the first variations of $\mathcal{L}$ with respect to its independent variables $\mathbf{q}, \tilde{\mathbf{q}}, \mathbf{q}_{0}, \tilde{\mathbf{q}_{0}}$ have to be set to zero, thus yielding the following set of equations:

$$
\begin{gathered}
{\left[\frac{d}{d t}-\mathbf{L}\right] \mathbf{q}=0,} \\
{\left[\frac{d}{d t}+\mathbf{L}^{H}\right] \tilde{\mathbf{q}}=0,} \\
\tilde{\mathbf{q}_{0}}=\frac{2}{\left\|\mathbf{q}_{0}\right\|^{2}} \mathbf{q}(T), \\
\mathbf{q}_{0}=\frac{\left\|\mathbf{q}_{0}\right\|^{4}}{2\|\mathbf{q}(T)\|^{2}} \tilde{\mathbf{q}}(0) .
\end{gathered}
$$

Equations (19a) and (19b) represent the direct and adjoint problem, respectively, while Eqs. (20a) and (20b) give the optimality conditions. An iterative solution procedure is applied to solve this set of equations. During each cycle of this procedure, a given initial condition is used to integrate the direct equation (19a) over the chosen time interval from $t=0$ to $t=T$ (forward). The output of this integration at time $t=T$ is $\mathbf{q}(T)$. This integration provides also a terminal condition $\tilde{\mathbf{q}}(T)$ for the adjoint equation (19b) which is subsequently integrated from $t=T$ to $t=0$ (backward) to produce $\tilde{\mathbf{q}}(0)$. From $\tilde{\mathbf{q}}(0)$ a new initial condition $\mathbf{q}(0)=\tilde{\mathbf{q}}(0)$ for the direct problem is determined [26]. The iterative process terminates when an appropriate convergence criterion is satisfied (i.e., the relative error of the growth function at the end of each iteration is less than $10^{-3}$ ).

For time-independent system matrices $(\delta=0)$, there is a more straightforward technique: the singular value 
decomposition (SVD). It evaluates the growth function as $\hat{G}(t)=s^{2}\left[\mathbf{V} \exp (t \boldsymbol{\Lambda}(\mathbf{L})) \mathbf{V}^{-1}\right]$, where $s\{\cdot\}$ denotes the maximum singular value of its matrix argument, $\mathbf{V}$ is the matrix whose columns are the eigenvectors of $\mathbf{L}$, and $\Lambda$ is the diagonal matrix containing the eigenvalues of $\mathbf{L}$.

\section{RESULTS}

The problem under investigation is characterized by three hydraulic parameters $\left(F_{0}, J, d_{s}\right)$, the wave number $k$, and three parameters describing the flow unsteadiness $(\omega, \delta, \phi)$. For simplicity, the domain of existence of morphodynamic instabilities is evaluated in the $F_{0}-k$ plane for fixed values of the remaining parameters. The dimensionless wave-number ranges in the interval $[0,1.5]$, since higher values run counter to the shallow water hypothesis. The parameter $F_{0}$ is taken in the range $[0.3,3]$ which covers both the dunes $\left(F_{0}<1\right)$ and antidunes $\left(F_{0}>1\right)$ regime. We also set $d_{s}=0.001$, $J=0.005$, and $\delta=Q_{0} / 4$ (other choices of $d_{s} \in\left[10^{-3}, 10^{-2}\right]$, $J \in\left[10^{-3}, 10^{-2}\right], \delta \in[0.1,0.9] Q_{0}$ would influence the results quantitatively but not qualitatively). Finally, the frequency $\omega$ and the phase $\phi$ will be varied in order to explore their influence on the system behavior.

We first investigate the role played by the flow unsteadiness on the asymptotic stability of the bed-stream system. To this end, periodic changes occurring on a time scale typical of sediment transport (daily or monthly variations) are considered. Figure 4 shows the marginal stability curve (corresponding to $v_{j}=1$ ), which divides the asymptotically stable and unstable regions, under steady and unsteady conditions and for different values of the phase $\phi$ in the $F_{0}-k$ plane. The Floquet multipliers do not depend on the frequency $\omega$, as also pointed out by [35] for bar instabilities. On the other hand, a weak dependence on the phase can be observed: the instability region enlarges with an increase in $\phi$. However, it is evident that the unsteadiness does not significantly affect the asymptotic fate of disturbances, and the instability regions are nearly unaltered when compared to the steady case. We remark that the instability region corresponds to an amalgam of two different modal processes: based on antidunes and roll waves. The

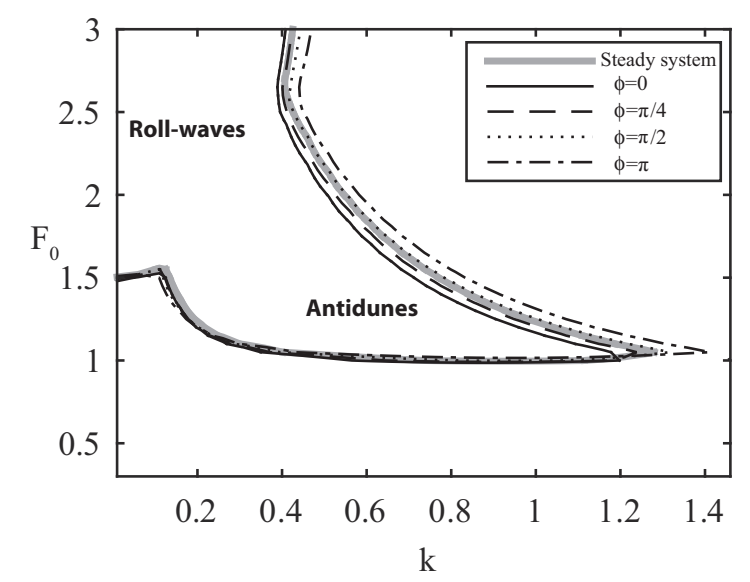

FIG. 4. The marginal stability curve $\left(v_{j}=1\right)$ is displayed for the steady (thick line) and the unsteady cases in the $F_{0}-k$ plane. The unsteady curves are obtained for $\omega=1$ and different values of $\phi$.

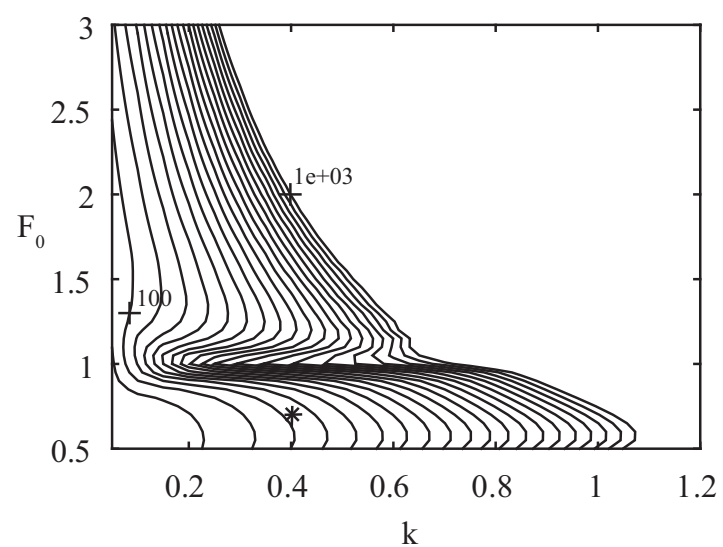

FIG. 5. Contour plot of optimal energy growth evaluated by the SVD for $\delta=0$ (steady case) in the $F_{0}-k$ plane. The line spacing is $\Delta \hat{G}=50$. The asterisk symbol marks the conditions chosen for the subsequent analysis.

latter prevail at low wave numbers and high Froude numbers (see Fig. 4) and arise mathematically, provided the temporal derivatives of Eqs. (1) and (2) are retained. In contrast, a third mode of instability (stemming from dune formation) is not detected asymptotically by this model, as highlighted in [36].

We now proceed to the transient growth analysis, focusing on intermediate time horizons. For time-independent baseflow conditions $(\delta=0)$, the optimal energy growth can be evaluated by the SVD and is reported in Fig. 5. Maximum amplification of initial energy is significant throughout the entire parameter space-an indication of substantial nonnormality of the operator $\mathbf{L}$. This finding also occurs in regions of asymptotic stability corresponding to dune formation and establishes the possibility of total energy growth by several orders of magnitude before exponential decay ultimately sets in. Noticeable transient growth of asymptotically stable modes is therefore expected during the early stages of bedform inception. This result also illustrates that the present onedimensional model is capable of predicting dune inception, albeit via transient effects.

By the nonmodal analysis, we have shown that river bedform dynamics exhibits remarkable transient growth, even for asymptotically stable wave numbers. The application of a nonmodal approach is critical in order to predict the inception of short-time instabilities not captured by the classical modal analysis. Another interesting point is the dominant bedform wavelength that is predicted by a nonmodal analysis. Figures 6(a) and 7(a) depict the dependence of the growth function $\hat{G}$ on the wave number $k$ for fixed optimization times for a dune and antidune case, respectively. The value of the wave number corresponding to the highest growth rate, $k_{\max }$, is reported in Figs. 6(b) and 7(b) as a function of time in conjunction with the corresponding values of the growth function, $\hat{G}_{\max }$. The wave number displaying the highest growth rate decreases with increasing time; this decay is more pronounced in the dune case (where the wave number drops from $k \simeq 1.5$ for $t=500$ to $k \simeq 0.2$ for $t=1.5 \times 10^{4}$ ) than in the antidune case (where the range of variation is rather limited, from $k \simeq 0.88$ for $t=500$ to $k \simeq 0.77$ for $t=1.5 \times 10^{4}$ ). 
(a)

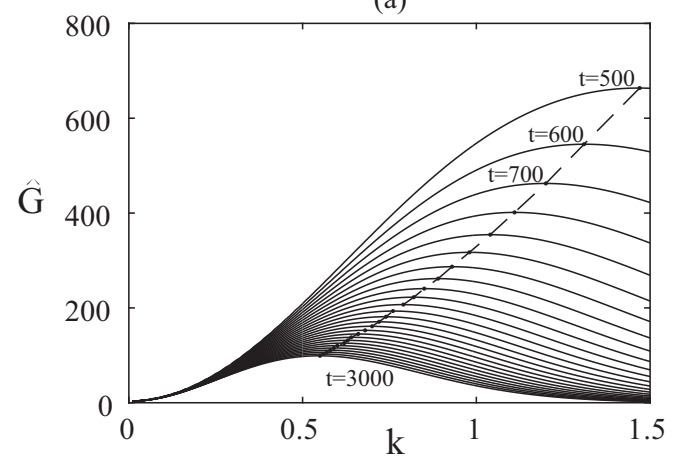

(b)

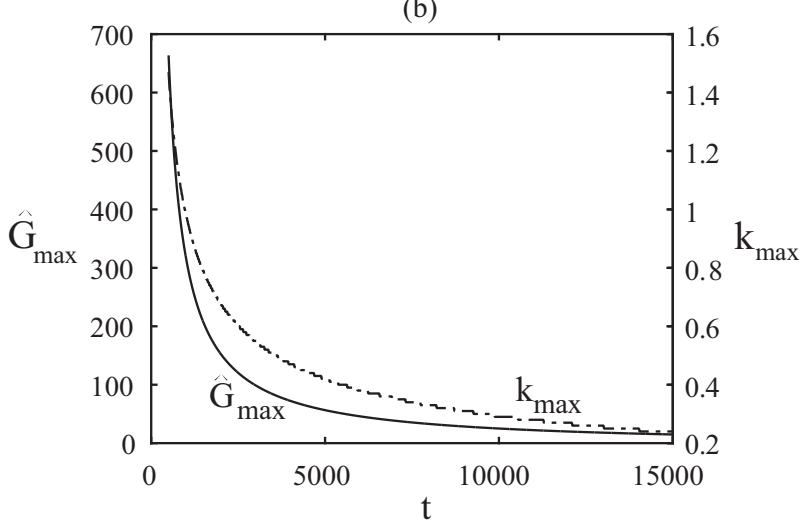

FIG. 6. Dune case $(\mathrm{Fr}=0.7)$ : (a) behavior of $\hat{G}$ as a function of the wave number $k$ for fixed times. The dashed line indicates the most unstable wave number for each time. The line spacing is $\Delta t=100$. (b) Evolution of the most unstable wave number over time in conjunction with the corresponding value of the growth function.

These results confirm a marked difference in behavior between the dunes and the antidunes. In the dune case, the wave number undergoes a significant decrease, i.e., the selected wavelength evolves in time, as already discussed in theoretical studies [31] and shown in experimental work [43]. Therefore, the dynamical system shows (at least in its linear behavior) different dominant wave numbers at different times: the wavelengths corresponding to transient growth will arise first, but will gradually disappear (the wavelength increases, but the amplitude decreases in time) since they are stable in the long term. What we discussed until now suggests the possibility of a linear scale selection mechanism for the dunes driven by nonmodal effects [31]. A distinct rise of a characteristic wavelength is not observable in the antidune case. These river bedforms are, in fact, selected by purely linear mechanisms, and the correct formation of these patterns can be predicted asymptotically. This entails that the asymptotic wavelength is selected immediately. Therefore, the dynamical system displays a characteristic wavelength that is selected at short times and approximately maintained for subsequent times; in other words, asymptotically unstable wave numbers already dominate the dynamics in the early stages. Finally, the results depicted in Figs. 6 and 7 show that the wavelengths favored and selected by the transient dynamics are attributable to typical wavelengths of dunes and antidunes (e.g., 1-50 m). For this reason, it can be stated that the bedforms which are
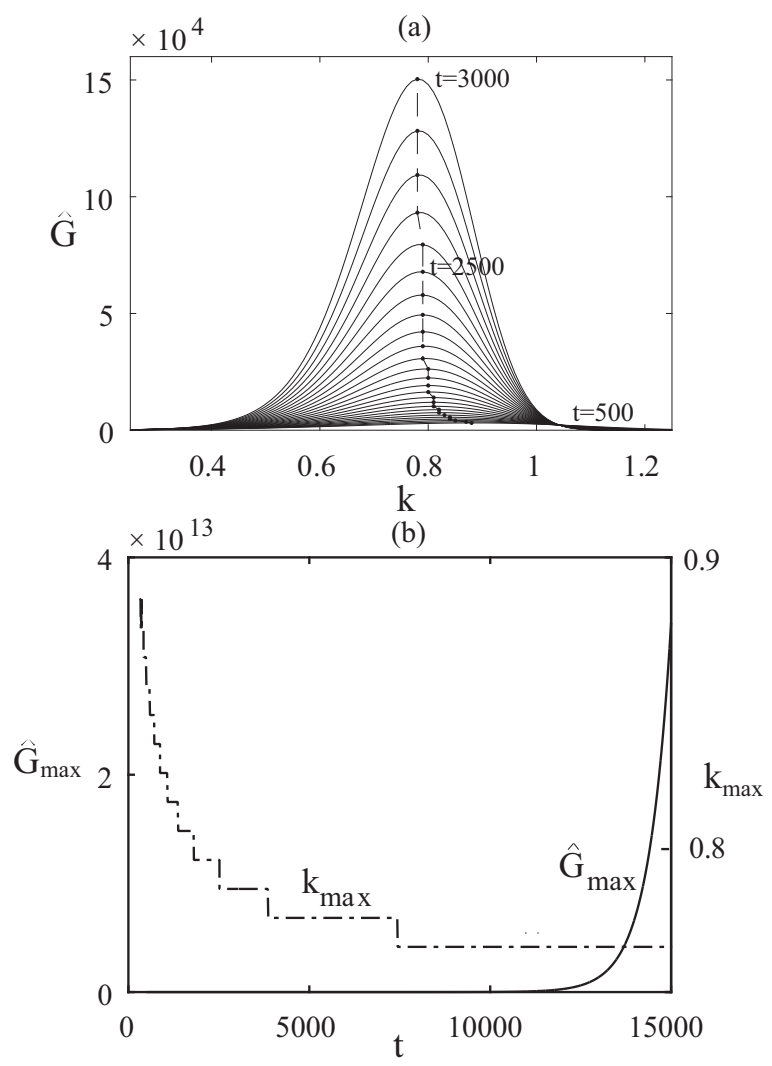

FIG. 7. Antidune case $(\mathrm{Fr}=1.2)$ : (a) behavior of $\hat{G}$ as a function of the wave number $k$. The dashed line indicates the most unstable wave number for each time. The line spacing is $\Delta t=100$. (b) Evolution of the most unstable wave number over time in conjunction with the corresponding value of the growth function.

transiently observed represent characteristic patterns of a river environment.

So far, the transient growth of bedforms has been investigated under the hypothesis of a constant flow velocity. Since the system has demonstrated the potential for remarkable transient growth under these steady conditions, it is interesting to next explore the effect of flow unsteadiness. To this end, the nonmodal analysis is extended to time-dependent flow discharge. The introduction of this temporal dependence requires the application of a variational approach based on adjoint techniques.

For addressing the influence of unsteady conditions, a representative point is chosen in the parameter region of dune formation $\left(F_{0}=0.7\right.$ and $\left.k=0.4\right)$. For a first analysis, the frequency of the oscillating base flow is changed, but the phase relation between the unsteady forcing and the response in the perturbation of the unforced system is held constant $(\phi=0)$. Figure 8 shows the growth function versus time for the steady case and for different values of $\omega$ for the unsteady case. In accordance with previous work on morphological pattern instabilities [30,31], the temporal evolution of $\hat{G}(t)$ shows an oscillatory structure. This is linked to the imaginary part of the least stable eigenvalue. The period of this oscillatory structure, $T_{f}=38$, is in fact an intrinsic property of the system; it is the inverse of its natural frequency $\omega_{f}$. As mentioned in the model section, the time scale emerging from the scaling is the 


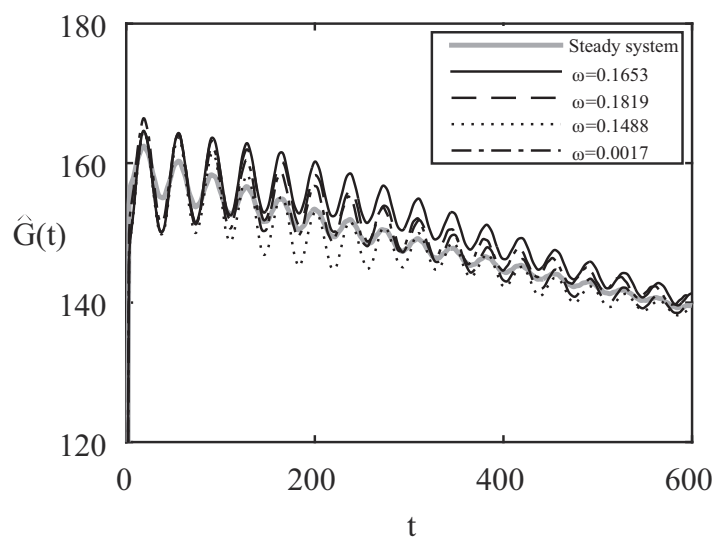

FIG. 8. Growth function versus time, evaluated at the point $\left\{k, F_{0}\right\}=\{0.4,0.7\}$ for the steady system and for different values of the frequency $\omega(\phi=0)$.

hydrodynamic one, thus the dimensionless frequency is given by $\omega=\omega^{*} \bar{D}_{0}^{*} / \bar{U}_{0}^{*}$.

The evaluation of the growth function by the direct-adjoint procedure for a wide range of time horizons is computationally demanding and usually unnecessary. All unsteady curves are instead obtained by the following simplified procedure [44]: (i) the growth function $\hat{G}(t)$ is computed by the more efficient SVD method for the corresponding steady problem; (ii) the time $t_{\max }$ at which $\hat{G}(t)$ reaches a maximum is identified; (iii) the iterative direct-adjoint technique is applied for $t=t_{\max }$ to determine the optimal initial condition $\mathbf{q}_{0}$; (iv) the direct problem (19a) is integrated in time starting from the optimal initial condition and the growth function is evaluated.

Our focus is on the role of the frequency $\omega$ in triggering a parametric resonance. Resonance occurs when the system is subjected to a periodic forcing with a frequency close to the natural oscillation of the system $\left(\omega_{f}=2 \pi / T_{f} \simeq 0.165\right)$. The curve obtained for $\omega=\omega_{f}=0.165$ (solid black line) shows a larger energy amplification than the steady case. Higher and lower values $\left(\omega=1.1, \omega_{f}=0.182\right.$ and $\left.\omega=0.9, \omega_{f}=0.149\right)$ result in an initial amplification followed by a smaller response for larger times.

According to the adopted scaling, the dimensionless time is given by $t=t^{*} \bar{U}_{0}^{*} / \bar{D}_{0}^{*}$. As the ratio $\bar{U}_{0}^{*} / \bar{D}_{0}^{*}$ is generally $\simeq 1$, we obtain $t \simeq t^{*} \mathrm{~s}$. It is interesting to observe the behavior of the system, when the period of the unsteadiness is similar to the characteristic time of river bedform formation-so as to probe the geomorphological relevance of our results. The dashed-dotted curve in Fig. 8 is obtained for $\omega=$ $\omega_{f} / 100=0.0017$, from which we determine $T^{*} \simeq 1 \mathrm{~h}$, and with an optimal condition computed at $t=t_{\max }$. It can be observed that the previous results are confirmed, even when periodic forcing with more physically significant frequencies, corresponding to longer times, are considered $(\omega=0.0017)$. Another remarkable result is related to the time necessary for the growth function to decay below unity (when the system returns to the base state). Extrapolating from the plot, the decay time appears to be $\simeq 2 \times 10^{4} \mathrm{~s} \simeq 6 \mathrm{~h}$, which is comparable to typical morphodynamic times and hence physically significant [45].

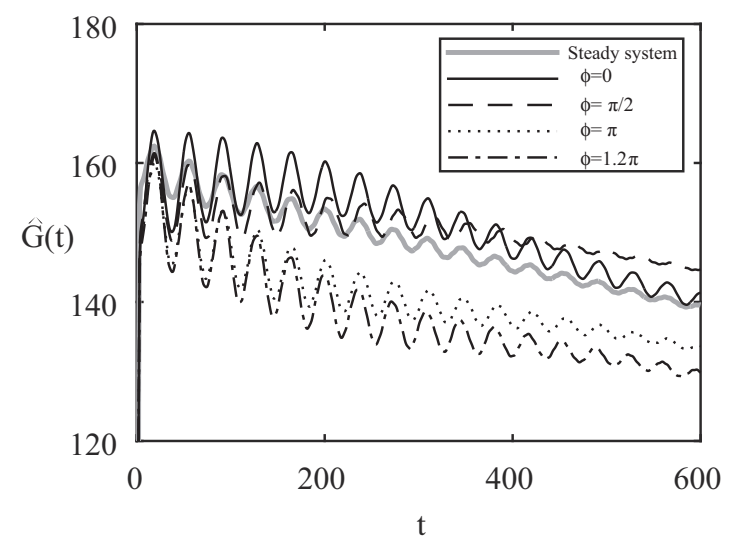

FIG. 9. Growth function versus time evaluated at the point $\left\{k, F_{0}\right\}=\{0.4,0.7\}$ for the steady system and for different values of the phase $\phi(\omega=0.1653)$.

Finally, Fig. 9 depicts the effect of the phase lag $\phi$. The different curves are obtained for a constant value of the frequency $(\omega=0.1653)$ and for phase values increasing from $\phi=0$ to $\phi=1.2 \pi$. We notice that an increase in $\phi$ produces a decrease of the maximum amplification of the system energy.

The obtained results demonstrate that the mutual interaction between flow unsteadiness and non-normality plays an important role in the stability of river bedforms. First, the nonmodal analysis of a one-dimensional model allowed us to demonstrate that bed perturbation can be transiently amplified by subcritical unsteady flows. Therefore, one of the mechanisms that contributes on the inception of dunes is linked to the transient amplification of disturbances. This result is beyond the reach of classical modal analysis, which focuses on the asymptotic disturbance behavior of the system only. Secondly, we have shown that flow unsteadiness can amplify, to a great extent, the transient growth of these perturbations when compared to equivalent steady conditions. Critical parameters governing this amplification effect are the frequency and the phase of the periodic forcing.

\section{CONCLUSIONS}

In the present work, we have addressed the interplay between unsteadiness and non-normality as it relates to the inception of river bedforms. Specifically, modal and nonmodal stability analyses have been carried out to investigate the riverbed instability and to assess the occurrence of transient growth. Both analyses have been performed while retaining all time dependencies of the system's governing equations. River bedform dynamics exhibit a significant amount of non-normality over a large part of parameter space. Consequently, substantial transient growth, despite asymptotically stable modes, is possible during the early stages of bed instabilities. It has been observed that the characteristic wavelength of the river bedform decreases with time; furthermore, it is correctly selected also for short time. The phase and frequency of the base-flow discharge have been recognized to play a key role in defining the magnitude of the transient energy amplifications. Importantly, the time scales of bedform transient growth are comparable to typical fluvial morphological and hydrological time scales, suggesting the development of bedforms by transient 
processes in real rivers. Finally, we have shown that our onedimensional antidune model is capable of capturing, modeling, and predicting the transient formation of river dunes.

Nonmodal analysis for unsteady conditions has revealed important results for the stability of subaqueous dunes. In view of this, it could be useful to apply a similar analysis for other morphological instabilities in which flow unsteadiness plays an important role, such as aeolian dunes [10]. As the barchan shape transition in the aeolian dune environment is very relevant, a nonmodal analysis could be used for improving existing studies on this transversal instability. The investigation of a transient-growth potential in this field is in fact lacking.

\section{APPENDIX: PARAMETERS OF THE MECHANISTIC SEDIMENT TRANSPORT MODEL}

The correction coefficients to account for gravity appearing in Eqs. (5), (6), (8), and (9) are here defined

$$
r_{s c}=\cos \alpha\left(1+\frac{\tan \alpha}{\mu}\right), \quad r_{d c}=\cos \alpha\left(1+\frac{\tan \alpha}{\mu_{d}}\right),
$$

$$
\begin{gathered}
r_{\mu}=1+\frac{\tan \alpha}{\mu}-\frac{\tan \alpha}{\mu_{d 0}}, \quad r_{s}=\left(r_{\mu} r_{d c}\right)^{-1 / 2}, \\
r_{e}=\left[1+\left(1-r_{\mu} r_{d c}\right) K_{0}\right]^{-3 / 2}, \quad r_{\lambda}=\left(\frac{r_{s c}}{r_{d c}}\right)^{1 / 2}, \\
\mu_{d}=\mu_{d 0} r_{\mu}, \\
T_{\mu}=T_{0} \frac{r_{\mu}}{r_{\lambda}^{2}}, \quad R_{\mu}=R_{0} r_{\mu} r_{d c}, \quad S_{\mu}=S_{0} \frac{r_{\mu}}{r_{\lambda}^{2}},
\end{gathered}
$$

with $K_{0}=\left(\mu_{d o} A_{e} / A_{s}\right)^{2 / 3}, \mu_{d 0}=0.3$ and $\mu=0.6 ; T_{0}, R_{0}$ and $S_{0}$ are the parameters for flat-bed conditions defined as

$$
\begin{gathered}
T_{0}=\frac{F_{0}^{2} \mu_{d 0}(1-p)}{\lambda^{2} \theta_{c h} f^{2} d_{s}^{2} R}, \\
R_{0}=\frac{R \mu_{d 0}}{F_{0}^{2}(R+1)}, \quad S_{0}=\frac{4 \mu_{d 0}\left(3 \lambda^{2} \theta_{c h} f^{2}\right)^{-1}}{c_{s} d_{s}(R+1)},
\end{gathered}
$$

with $R=1.65, \mu_{d 0}=0.3, c_{s}=4 / 3, \lambda=0.7, \theta_{c h}=0.047$, and $p=0.4$.
[1] W. J. Lillycrop, J. D. Rosati, and D. D. McGehee, A study on sand waves in the Panama City, Florida, entrance channel, Tech. Rep. No. CERC-TR-89-7 (U.S. Army Engineer and Development Center, 1989), http://acwc.sdp.sirsi.net/client/ search/asset/1001036.

[2] D. J. Harbor, J. Sediment. Res. 68, 750 (1998).

[3] P. Y. Julien and G. J. Klaassen, J. Hydraul. Eng. 121, 657 (1995).

[4] J. Best, J. Geophys. Res.: Earth Surf. 110, F04S02 (2005).

[5] A. I. Packman and N. H. Brooks, Water Resour. Res. 37, 2591 (2001).

[6] F. Boano, J. W. Harvey, A. Marion, A. I. Packman, R. Revelli, L. Ridolfi, and A. Wörman, Rev. Geophys. 52, 603 (2014).

[7] A. Blom, J. S. Ribberink, and H. J. de Vriend, Water Resour. Res. 39, 1025 (2003).

[8] M. Kleinhans, Earth Sci. Rev. 65, 75 (2004).

[9] H. Niiya, A. Awazu, and H. Nishimori, Phys. Rev. Lett. 108, 158001 (2012).

[10] H. P. Melo, E. J. Parteli, J. S. Andrade, and H. J. Herrmann, Physica A: Stat. Mech. Appl. 391, 4606 (2012).

[11] L. Guignier, H. Niiya, H. Nishimori, D. Lague, and A. Valance, Phys. Rev. E 87, 052206 (2013).

[12] N. Endo, K. Taniguchi, and A. Katsuki, Geophys. Res. Lett. 31, L12503 (2004).

[13] D. McCulloch and R. Janda, J. Sediment. Res. 34, 694 (1964).

[14] R. Vesipa, River antidunes and bars: New models and nonmodal analysis, Ph.D. thesis, Politecnico di Torino, 2013.

[15] F. Engelund, J. Fluid Mech. 42, 225 (1970).

[16] K. J. Richards, J. Fluid Mech. 99, 597 (1980).

[17] C. DiCristo, M. Iervolino, and A. Vacca, J. Hydraul. Res. 44, 480 (2006).

[18] M. Colombini, J. Fluid Mech. 502, 1 (2004).

[19] M. Colombini and A. Stocchino, J. Fluid Mech. 695, 63 (2012).

[20] B. Andreotti, P. Claudin, O. Devauchelle, O. Durán, and A. Fourrière, J. Fluid Mech. 690, 94 (2012).
[21] P. J. Schmid and D. S. Henningson, Stability and Transition in Shear Flows, 1st ed. (Springer-Verlag, Berlin, 2001).

[22] D. Rempfer, Annu. Rev. Fluid Mech. 35, 229 (2003).

[23] C. Lee and J. Wu, Appl. Mech. Rev. 61, 030802 (2008).

[24] L. N. Trefethen, A. E. Trefethen, S. C. Reddy, and T. A. Driscoll, Science 261, 578 (1993).

[25] K. M. Butler and B. F. Farrell, Phys. Fluids A 4, 1637 (1992).

[26] P. J. Schmid, Annu. Rev. Fluid Mech. 39, 129 (2007).

[27] S. C. Reddy and D. S. Henningson, J. Fluid Mech. 252, 209 (1993).

[28] P. J. Olsson and D. S. Henningson, Stud. Appl. Math. 94, 183 (1995).

[29] S. V. Malik and A. P. Hooper, Phys. Fluids 19, 052102 (2007).

[30] C. Camporeale and L. Ridolfi, Water Resour. Res. 45, W08418 (2009).

[31] C. Camporeale and L. Ridolfi, Phys. Fluids 23, 104102 (2011).

[32] R. Vesipa, C. Camporeale, and L. Ridolfi, Europhys. Lett. 100, 64002 (2012).

[33] M. Tubino, R. Repetto, and G. Zolezzi, J. Hydraul. Res. 37, 759 (1999).

[34] F. Visconti, C. Camporeale, and L. Ridolfi, J. Geophys. Res. 115, F04042 (2010).

[35] P. Hall, J. Fluid Mech. 499, 49 (2004).

[36] R. Vesipa, L. Ridolfi, and C. Camporeale, Phys. Fluids 24, 094104 (2012).

[37] C. Camporeale, C. Canuto, and L. Ridolfi, Theor. Comput. Fluid Dyn. 26, 51 (2012).

[38] G. Seminara, L. Solari, and G. Parker, Water Resour. Res. 38, 31 (2002).

[39] G. Parker, G. Seminara, and L. Solari, Water Resour. Res. 39, 1183 (2003).

[40] R. F. Dressler, J. Hydraul. Res. 16, 205 (1978).

[41] S. Wright and G. Parker, J. Hydraul. Eng. 130, 796 (2004). 
[42] D. P. G. Foures, C. P. Caulfield, and P. J. Schmid, Phys. Rev. E 86, 026306 (2012).

[43] S. E. Coleman and B. W. Melville, J. Hydraul. Eng. 122, 301 (1996).
[44] C. Camporeale and P. J. Schmid, J. Fluid Mech. 768, 524 (2015).

[45] P. P. Jansen, L. Van Bendegom, J. Van den Berg, M. De Vries, and A. Zanen, Principles of River Engineering: The Non-Tidal Alluvial River (Delftse Uitgevers Maatschappij, Delft, 1994). 